\title{
Monitoring the Conformation of the Sba1/Hsp90 Complex in Presence of Nucleotides with Mn(II)-Based Double Electron-Electron Resonance
}

\author{
Angeliki Giannoulis ${ }^{1}$, Akiva Feintuch ${ }^{1}$, Tamar Unger ${ }^{2}$, Shiran Amir ${ }^{2}$, Daniella Goldfarb ${ }^{1}$ \\ ${ }^{1}$ Department of Chemical and Biological Physics, Weizmann Institute of Science, Rehovot, 76100, \\ Israel
}

\footnotetext{
${ }^{2}$ Structural Proteomics Unit, Department of Life Sciences Core Facilities, Weizmann Institute of Science, Rehovot, 76100, Israel

*Corresponding author: daniella.goldfarb@weizmann.ac.il
}

\section{Supporting information}

\section{Contents}

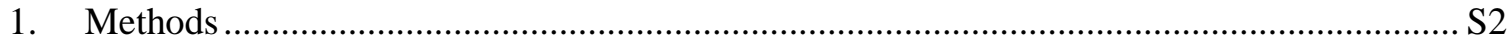

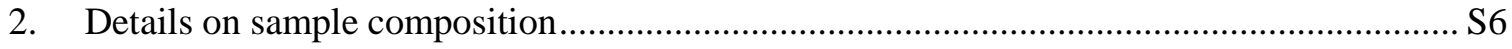

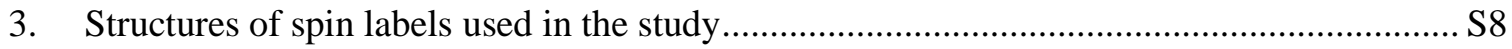

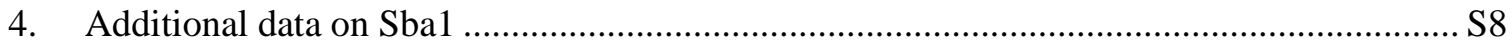

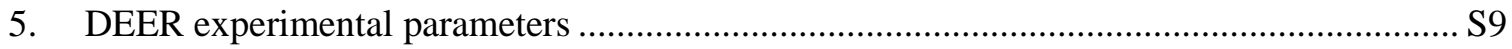

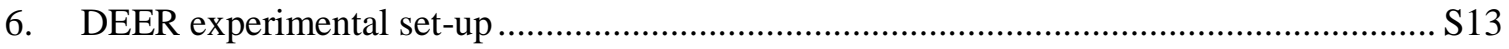

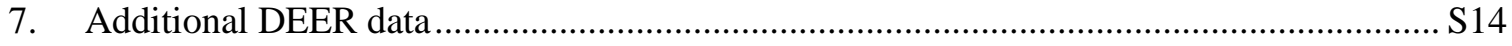

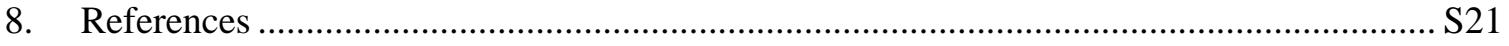




\section{Methods}

Protein expression and purification. The Hsp90 co-chaperone Sba1 construct with an N-terminal His $_{6}$ tag and a TEV cleavage site (pET28a-TEVH) was transformed into BL21(DE3) chemically competent cells and plated on lysogeny broth (LB)-agar plates containing $50 \mu \mathrm{g} / \mathrm{mL}$ kanamycin (kan) using standard protocols. A single colony was transferred for growth in $10 \mathrm{~mL} \mathrm{LB}$ with 30 $\mu \mathrm{g} / \mathrm{mL}$ kan overnight at $37^{\circ} \mathrm{C}, 250 \mathrm{rpm} .1 \mathrm{~mL}$ of the culture was diluted to $50 \mathrm{~mL} \mathrm{LB}$ and used for growth at $37^{\circ} \mathrm{C}, 250 \mathrm{rpm}$ to an $\mathrm{OD}_{600} 0.6-0.8$ before expression was induced by $0.5 \mathrm{mM}$ isopropyl $\beta$-D-1-thioogalactopyranoside (IPTG) at $37{ }^{\circ} \mathrm{C}, 250 \mathrm{rpm}, 4 \mathrm{~h}$. The cells were pelleted by centrifugation (4300 rpm, $10 \mathrm{~min})$ and suspended in sonication buffer $(50 \mathrm{mM}$ Tris $\cdot \mathrm{HCl}, 500 \mathrm{mM}$ $\mathrm{NaCl}, \mathrm{pH}$ 8.0) supplemented with $1 \mu \mathrm{l} / \mathrm{ml}$ protease inhibitor cocktail (Calbiochem Set III, EDTAfree) and $1 \mathrm{mM}$ phenylmethylsulfonyl fluoride (PMSF). Cell lysis was performed on ice by sonication (12 cycles, 100\% amplitude) and the cellular debris was separated by centrifugation (13000 rpm, $15 \mathrm{~min}$ ) from the protein-containing supernatant. To the supernatant Ni-NTA beads pre-equilibrated with sonication buffer were added and rotated gently at $4{ }^{\circ} \mathrm{C}$ for $1 \mathrm{~h}$, followed by centrifugation at $2000 \mathrm{rpm}, 1 \mathrm{~min}$. Following removal of the unbound proteins, the beads were washed twice with wash buffer (50 $\mathrm{mM} \mathrm{NaH}_{2} \mathrm{PO} 4 \mathrm{pH} 8.0,300 \mathrm{mM} \mathrm{NaCl}, 20 \mathrm{mM}$ imidazole $)$ and centrifuged at $2000 \mathrm{rpm}, 1 \mathrm{~min}$. The protein was eluted from the beads using elution buffer $(50 \mathrm{mM}$ $\mathrm{NaH}_{2} \mathrm{PO} 4 \mathrm{pH} 8.0,300 \mathrm{mM} \mathrm{NaCl}, 500 \mathrm{mM}$ imidazole). The eluted Sbal was of high purity as visually judged from SDS-PAGE with $12 \%$ acrylamide. The buffer was exchanged to $20 \mathrm{mM}$ deuterated Tris $\mathrm{HCl}$ buffer, $20 \mathrm{mM} \mathrm{KCl,} 20 \%$ glycerol- $d_{8}$, pD 7.4 via continuous exchange with Vivaspin $500 \mu \mathrm{L}$ concentrator with molecular cut-off $3 \mathrm{kDa}$. The protein was stored at $-80{ }^{\circ} \mathrm{C}$. yHsp90 expression and purification protocol is detailed in ref ${ }^{1}$.

Spin labeling and sample preparation. Nitroxide labeling: 1 equiv. of $3,3^{\prime}, 3^{\prime \prime}$ phosphanetriyltripropanoic acid (TCEP, in MQ) was added to A152C yHsp90 or WT Sba1 (in 20 $\mathrm{mM}$ Tris $\mathrm{HCl}, 20 \mathrm{mM} \mathrm{KCl}, 20 \%$ glycerol, $\mathrm{pH}$ 7.4) and the solution was left to react for $0.5 \mathrm{~h}$. TCEP was removed with Micro Bio-Spin ${ }^{\mathrm{TM}}$ P-6 pre-equilibrated with $20 \mathrm{mM}$ Tris $\mathrm{HCl}$ buffer, $20 \mathrm{mM}$ 
$\mathrm{KCl}, \mathrm{pH}$ 7.4. Immediately after, MTSL or proxyl (in $\mathrm{EtOH}, \mathrm{pH} 7.0,50 \mathrm{mM}$, see structures in SI, Fig. S1)) was added in 10-fold excess to WT Sba1 or A152C yHsp90, respectively, and the solution was stirred overnight at $4{ }^{\circ} \mathrm{C}$. The excess of label was removed with Vivaspin $500 \mu \mathrm{L}$ concentrator with molecular cut-off $30 \mathrm{kDa}$ for Hsp90 or $3 \mathrm{kDa}$ for Sba1 via continuous exchange with the EPR buffer (20 mM deuterated Tris $\mathrm{HCl}$ buffer, $20 \mathrm{mM} \mathrm{KCl,} 20 \%$ glycerol- $d_{8}, \mathrm{pD}$ 7.4)._Mn(II) labeling: First, a solution of $\mathrm{MnCl}_{2} 6 \mathrm{H}_{2} \mathrm{O} /$ nucleotide was freshly prepared at 1/1 ratio (in MQ) and the $\mathrm{pH}$ was adjusted to 7.0 with $1 \mathrm{M} \mathrm{NaOH}$ aq. solution. The solution was then added to yHsp90 (WT or A152C/NO in $20 \mathrm{mM}$ deuterated Tris $\mathrm{HCl}$ buffer, $20 \mathrm{mM} \mathrm{KCl}, 20 \%$ glycerol- $d_{8}, \mathrm{pD}$ 7.4). In samples that contained Sba1 (labeled or non-labeled), Sba1 (in $20 \mathrm{mM}$ deuterated Tris $\mathrm{HCl}$ buffer, $20 \mathrm{mM} \mathrm{KCl}, 20 \%$ glycerol- $d_{8}, \mathrm{pD}$ 7.4) was added after the addition of $\mathrm{Mn}(\mathrm{II})$-nucleotide in slight excess with respect to yHsp90. All samples were frozen after further 2 min unless otherwise stated. For samples composition see Table S1 in SI. Gd(III) labeling of yHsp90 mutants is detailed in ref ${ }^{1}$. EPR spectroscopy. CW-EPR data were recorded at X-band $(9.4 \mathrm{GHz})$ at room temperature. All other data were recorded on a home-built W-band $(94.9 \mathrm{GHz})$ spectrometer equipped with a $2 \mathrm{~W}$ solid state amplifier from Quinstar Inc ${ }^{2}$. W-band data were recorded at cryogenic temperatures and the temperature was stabilized with a cryo-free cooling system from ColdEdge Technologies.

Echo-detected EPR (ED-EPR). ED-EPR spectra were recorded with a Hahn echo sequence ( $\pi / 2$ $-\tau-\pi-$ echo) and sweeping the magnetic field. For samples containing only NO spins the EDEPR spectra were recorded at $25 \mathrm{~K}$ using microwave (mw) pulse lengths $\pi / 2=20 \mathrm{~ns}, \pi=40 \mathrm{~ns}$, interpulse delay $\tau=550 \mathrm{~ns}$ and repetition time $20 \mathrm{~ms}$. For samples containing both $\mathrm{Mn}(\mathrm{II})$ and NO spins the ED-EPR spectra were recorded at $10 \mathrm{~K}$ and are shown optimized for the NO spins and were recorded using the same parameters as for the samples having only NO spins. For samples containing only $\mathrm{Mn}(\mathrm{II})$ or $\mathrm{Gd}(\mathrm{III})$ the ED-EPR spectra were recorded at $10 \mathrm{~K}$ using $\pi / 2=15 \mathrm{~ns}$, $\pi=30 \mathrm{~ns}$, inter-pulse delay $\tau=550 \mathrm{~ns}$ and repetition time $0.8 \mathrm{~ms}$. 
Echo decay traces were recorded at the maximum of the NO EPR spectrum and at the $4^{\text {th }}$ EPR hyperfine component of the $\mathrm{Mn}(\mathrm{II})$ spectrum monitoring the echo intensity of a Hahn echo sequence by varying the $\tau$ interval using pulse lengths and repetition times as above.

${ }^{31} \mathbf{P}$ W-band ENDOR shown in Fig. S5D (black) was recorded at $10 \mathrm{~K}$ on the $4^{\text {th }} \mathrm{Mn}$ (II) line using the standard Davies ENDOR sequence $\left(\pi_{\mathrm{mw}}-\mathrm{T}-t_{\mathrm{rf}}-\mathrm{T}-\pi / 2_{\mathrm{mw}}-\tau-\pi_{\mathrm{mw}}-\tau-\text { echo }\right)^{3}$ combined with a Carr Purcell Meiboom Gill (CPMG) sequence for enhanced sensitivity ${ }^{4}$. In this experiment the echo intensity is measured as a function of the frequency of the rf pulse which was swept from 52 to $64 \mathrm{MHz}$ with $50 \mathrm{kHz}$ step using random acquisition ${ }^{5}$. An eight-step phase cycling was applied. The microwave pulse lengths were $\pi / 2_{\mathrm{mw}}=100 \mathrm{~ns}, \pi_{\mathrm{mw}}=200 \mathrm{~ns}$, the inter-pulse delay was $\tau=750 \mathrm{~ns}$ and the repetition time was $4 \mathrm{~ms}$. The rf pulse length, $t_{\mathrm{RF}}$, was $40 \mu \mathrm{s}$ and $\mathrm{T}$ was $45 \mu \mathrm{s}$. The echoes were integrated using a home-made Matlab script and the data were plotted as a function of the swept $\mathrm{rf}$ range minus the ${ }^{31} \mathrm{P}$ Larmor frequency. The other ENDOR spectra given for comparison have been previously published in $\operatorname{ref}^{1}$.

W-band DEER. The data were recorded using the four-pulse $r$ DEER $\left(\pi / 2_{\text {vobs }}-\tau_{1}-\pi_{\text {vobs }}-\left(\tau_{1}-t\right)-\right.$ $\left.\pi_{\text {vpump }}-\left(\tau_{2}+\mathrm{t}\right)-\pi_{\mathrm{vobs}}-\tau_{2}-\mathrm{echo}\right)^{6}$ or the standard four-pulse DEER $\left(\pi / 2_{\text {vobs }}-\tau_{1}-\pi_{\text {vobs }}-\left(\tau_{1}+\mathrm{t}\right)-\right.$ $\pi_{\text {vpump }}-\left(\tau_{2}-\mathrm{t}\right)-\pi_{\text {vobs }}-\tau_{2}-$ echo $)$ sequence ${ }^{7}$ using chirp pump pulse $(s)^{8}$ monitoring the echo intensity with increasing t of the pump pulse $\pi_{v p u m p}$. An eight-step phase cycling was applied for both DEER and $r$ DEER sequences. The general set-up is given in SI Fig. S3 and all the parameters of the experiments are given in Tables S2-S7.

DEER data analysis. The primary DEER data were transformed into distance distributions using the DeerAnalysis $2018^{9}$ software and the Tikhonov regularization criterion. The background contributions to the primary DEER data were removed by fitting a background homogeneous to 3 dimensions or in cases where the DEER traces looked unphysical the background dimensionality was fitted (in the range of 3.0-3.5) in order to obtain reasonable fits in the frequency and time domain data. The regularization parameter was 100 for $\mathrm{Mn}$ (II)-NO and $\mathrm{Mn}$ (II)-Mn(II) data, and 
100-1000 for NO-NO data. The contributions of the background signal were evaluated within the validation tool of the DeerAnalysis 2018 program performed from 5\% to $80 \%$ of the DEER time trace in 16 trials, and white noise of level 1.5 was added in 10 trials. Only datasets within $15 \%$ of the best root mean square deviation were retained (i.e. default prune level 1.15) affording the confidence intervals of the plotted distance distributions. The color bars below the distance distributions denote reliability of the distance as follows: green= shape reliable; yellow= mean and width reliable; orange $=$ mean reliable; red $=$ non-reliable. For Fig. 2D, E they were calculated using the DEER trace with the shortest length.

Modeling of spin label rotamers and distance calculation. The possible rotameric positions of the MTSL anchored on Sba1 and of 3-maleimido-PROXYL anchored on A152C were calculated using the online version MtsslWizard ${ }^{10}$ software and the X-ray structure of yHsp90 in presence of AMP-PNP and Sba1 (PDB entry $2 \mathrm{CG}^{11}$ ). For Sba1/NO the parameters were 'loose' and for A152C/NO 'very loose', as these allowed insertion of $>5$ rotamers. The metal co-factor was inserted to the crystal structure as a pseudo-atom coordinated by the $\beta$ - and $\gamma$-phosphates of AMPPNP in each protomer ${ }^{12}$. The $\mathrm{Mn}$ (II)-Mn(II) distance was calculated using the distance measurement tool of PyMOL software between the two pseudo-atoms. The Mn(II)-NO distance distributions were calculated using a homemade MATLAB script which takes into account the position of all the rotamers of the NO label and the coordinates of each pseudo-atom. The histogram of the distance was smoothed using a Gaussian filter. The NO-NO distances were calculated directly in MtsslWizard taking into account all the rotamers of each NO site. 


\section{Details on sample composition}

Table S1. Samples composition.

\begin{tabular}{|c|c|c|c|c|c|c|c|}
\hline Sample & $\begin{array}{l}\text { Shown } \\
\text { in Fig. }\end{array}$ & $\begin{array}{l}\mathrm{C}_{\mathrm{Hsp} 90} / \\
\mu \mathrm{M}\end{array}$ & $\begin{array}{l}\mathrm{C}_{\text {Sbal }} / \\
\mu \mathrm{M}\end{array}$ & $\begin{array}{l}\mathrm{C}_{\text {Sba1.MTSL }} / \\
\mu \mathrm{M}\end{array}$ & $\begin{array}{l}\mathrm{M}(\mathrm{II}) \text { nucleotide } \\
\mu \mathrm{M}\end{array}$ & $\begin{array}{l}\text { Hsp90/Sba1/ } \\
\text { M (II) }\end{array}$ & $\begin{array}{lr}\text { DEER } & \text { experiment } \\
\text { (unless } & \text { otherwise } \\
\text { stated*) } & \end{array}$ \\
\hline $\begin{array}{l}\text { Sba1/NO+ } \\
\text { Mg(II) AMP- } \\
\text { PNP }\end{array}$ & $\begin{array}{l}\mathrm{S} 4, \\
\text { green }\end{array}$ & - & - & 310 & 4000 & $0 / 1 / 13$ & NO-NO \\
\hline $\begin{array}{l}\text { WT+Sba1/NO+ } \\
\text { Mg(II) AMP- } \\
\text { PNP }\end{array}$ & S4, black & 290 & - & 310 & 4000 & $1 / 1.1 / 13$ & NO-NO \\
\hline $\begin{array}{l}\text { WT+Sba1/NO+ } \\
\mathrm{Mg}(\mathrm{II}) \cdot \mathrm{ADP}\end{array}$ & S4, blue & 290 & - & 310 & 4000 & $1 / 1.1$ & NO-NO \\
\hline $\begin{array}{l}\text { WT+ } \\
\text { Sba1/NO+ } \\
\text { Mn(II)·AMP- } \\
\text { PNP }\end{array}$ & $\begin{array}{l}1 \mathrm{C}, \\
\text { black } \\
\text { and S6A }\end{array}$ & 140 & - & 177 & 128 & $1 / 1.3 / 0.9$ & $\begin{array}{l}\text { Mn(II)-NO, } \\
\text { Mn(II)-Mn(II) }\end{array}$ \\
\hline $\begin{array}{l}\text { WT+ } \\
\text { Sba1/NO } \\
+\mathrm{Mn}(\mathrm{II}) \cdot \mathrm{ADP}\end{array}$ & 1C, blue & 180 & - & 200 & 180 & $1 / 1.1 / 1$ & $\mathrm{Mn}(\mathrm{II})-\mathrm{NO}$ \\
\hline $\begin{array}{l}\text { WT+ } \\
\text { Sba1/NO } \\
+\mathrm{Mn}(\mathrm{II}) \cdot \mathrm{ATP}, \\
0.5 \mathrm{~h}\end{array}$ & S5B & 165 & - & 192 & 165 & $1 / 1.2 / 1$ & $\mathrm{Mn}$ (II)-NO \\
\hline $\begin{array}{l}\text { WT+Sba1+ } \\
\text { Mn(II) ATP, 3h }\end{array}$ & S5D & 192 & 253 & - & 180 & $1 / 0.9 / 1.3$ & ENDOR* \\
\hline $\begin{array}{l}\text { Sba1/NO+ } \\
\text { Mn(II)'AMP- } \\
\text { PNP }\end{array}$ & S6B & - & - & 177 & 128 & $0 / 1 / 0.9$ & $\mathrm{Mn}$ (II)-NO \\
\hline $\begin{array}{l}\text { D560C/Gd(III)+ } \\
\text { Mg(II) AMP- } \\
\text { PNP-Sba1 }\end{array}$ & S7 & 100 & - & - & 10000 & $1 / 0 / 100$ & Gd(III)-Gd(III) \\
\hline $\begin{array}{l}\text { D560C/Gd(III)+ } \\
\text { Mg(II) AMP- } \\
\text { PNP+Sba1 }\end{array}$ & S7 & 120 & 240 & - & 5000 & $1 / 2 / 42$ & Gd(III)-Gd(III) \\
\hline $\begin{array}{l}\text { K637C/Gd(III)+ } \\
\text { Mg(II) AMP- } \\
\text { PNP-Sba1 }\end{array}$ & S7 & 100 & - & - & 10000 & $1 / 0 / 100$ & Gd(III)-Gd(III) \\
\hline $\begin{array}{l}\text { K637C/Gd(III)+ } \\
\text { Mg(II) AMP- } \\
\text { PNP+Sba1 }\end{array}$ & S7 & 120 & 240 & - & 5000 & $1 / 2 / 42$ & Gd(III)-Gd(III) \\
\hline
\end{tabular}


Table S1 (continued). Samples composition.

\begin{tabular}{|c|c|c|c|c|c|c|c|}
\hline Sample & $\begin{array}{l}\text { Shown in } \\
\text { Fig. }\end{array}$ & $\begin{array}{l}\mathrm{C}_{\mathrm{Hsp} 90} / \\
\mu \mathrm{M}\end{array}$ & $\begin{array}{l}\mathrm{C}_{\text {Sbal }} / \\
\mu \mathrm{M}\end{array}$ & $\begin{array}{l}\mathrm{C}_{\text {Sbal.MTSL }} / \\
\mu \mathrm{M}\end{array}$ & $\begin{array}{l}\mathrm{M}(\mathrm{II}) \text { nucleotide / } \\
\mu \mathrm{M}\end{array}$ & $\begin{array}{l}\text { Hsp90/Sba1/ } \\
\text { M (II) }\end{array}$ & DEER experiment \\
\hline $\begin{array}{l}\text { A152C/NO } \\
+\mathrm{Mg}(\mathrm{II}) \cdot \mathrm{AMP}- \\
\text { PNP-Sba1 }\end{array}$ & 2B, blue & 150 & - & - & 10000 & $1 / 0 / 67$ & NO-NO \\
\hline $\begin{array}{l}\text { A152C/NO } \\
+\mathrm{Mg}(\mathrm{II}) \cdot \mathrm{AMP}- \\
\mathrm{PNP}+\mathrm{Sba} 1\end{array}$ & 2B, cyan & 150 & 300 & - & 10000 & $1 / 2 / 67$ & $\mathrm{NO}-\mathrm{NO}$ \\
\hline $\begin{array}{l}\text { A152C/NO } \\
+\mathrm{Mn}(\mathrm{II}) \text { AMP- } \\
\text { PNP-Sba1 }\end{array}$ & 2D, blue & 150 & - & - & 120 & $1 / 0 / 0.8$ & $\mathrm{Mn}(\mathrm{II})-\mathrm{NO}$ \\
\hline $\begin{array}{l}\text { A152C/NO } \\
+\mathrm{Mn}(\mathrm{II}) \cdot \mathrm{AMP}- \\
\mathrm{PNP}+\mathrm{Sba} 1\end{array}$ & $\begin{array}{l}\text { 2D, cyan } \\
\text { and } \\
\text { S9 rep }\end{array}$ & 118 & 237 & - & 118 & $1 / 2 / 1$ & $\mathrm{Mn}(\mathrm{II})-\mathrm{NO}$ \\
\hline $\begin{array}{l}\text { A152C/NO } \\
+\mathrm{Mn}(\mathrm{II}) \cdot \mathrm{AMP}- \\
\mathrm{PNP}+\mathrm{Sba} 1\end{array}$ & $\begin{array}{l}\text { S9, cyan } \\
\# 2\end{array}$ & 185 & 371 & & 148 & $1 / 2 / 0.8$ & $\mathrm{Mn}(\mathrm{II})-\mathrm{NO}$ \\
\hline $\begin{array}{l}\text { A152C/NO } \\
+\mathrm{Mn}(\mathrm{II}) \cdot \mathrm{ADP}- \\
\text { Sba1 }\end{array}$ & $\begin{array}{l}3 \mathrm{D} \text {, green } \\
\text { and } \mathrm{S} 9, \\
\text { green \#2 }\end{array}$ & 150 & - & - & 120 & $1 / 0 / 0.8$ & $\mathrm{Mn}(\mathrm{II})-\mathrm{NO}$ \\
\hline $\begin{array}{l}\text { A152C/NO } \\
+\mathrm{Mn}(\mathrm{II}) \cdot \mathrm{ADP}+ \\
\text { Sba1 }\end{array}$ & $\begin{array}{l}3 \mathrm{D}, \text { light } \\
\text { green }\end{array}$ & 200 & 262 & - & 250 & $1 / 1.3 / 1.2$ & $\mathrm{Mn}$ (II)-NO \\
\hline $\begin{array}{l}\text { A152C/NO } \\
+\mathrm{Mn}(\mathrm{II}) \cdot \mathrm{ADP}+ \\
\text { Sba1 }\end{array}$ & $\begin{array}{l}\text { S9, light } \\
\text { green \#2 }\end{array}$ & 200 & 262 & - & 360 & $1 / 1.3 / 1.8$ & $\mathrm{Mn}$ (II)-NO \\
\hline $\begin{array}{l}\text { WT+Mn AMP- } \\
\text { PNP+Sba1 }\end{array}$ & $\begin{array}{l}3, \quad \mathrm{~S} 10, \\
\text { cyan \#1 }\end{array}$ & 286 & 307 & - & 265 & $1 / 1.1 / 0.9$ & $\mathrm{Mn}(\mathrm{II})-\mathrm{Mn}(\mathrm{II})$ \\
\hline $\begin{array}{l}\text { A152+Mn:AM } \\
\text { P-PNP+Sba1 }\end{array}$ & $\begin{array}{l}3, \quad \mathrm{~S} 10, \\
\text { cyan \#2 }\end{array}$ & 218 & 263 & - & 218 & $1 / 1.2 / 1$ & $\mathrm{Mn}(\mathrm{II})-\mathrm{Mn}(\mathrm{II})$ \\
\hline $\begin{array}{l}\text { WT+Mn'ADP+ } \\
\text { Sba1 }\end{array}$ & $\begin{array}{l}3, \quad \mathrm{~S} 10, \\
\text { light green } \\
\# 1\end{array}$ & 200 & 262 & - & 250 & $1 / 1.3 / 1.2$ & $\mathrm{Mn}(\mathrm{II})-\mathrm{Mn}(\mathrm{II})$ \\
\hline $\begin{array}{l}\text { A152C+Mn'AD } \\
\text { P+Sba1 }\end{array}$ & $\begin{array}{l}3, \quad \text { S10, } \\
\text { light green } \\
\# 2\end{array}$ & 200 & 262 & - & 250 & $1 / 1.3 / 1.2$ & $\operatorname{Mn}(\mathrm{II})-\mathrm{Mn}(\mathrm{II})$ \\
\hline
\end{tabular}




\section{Structures of spin labels used in the study}

A

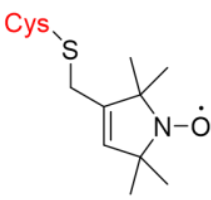

MTSL

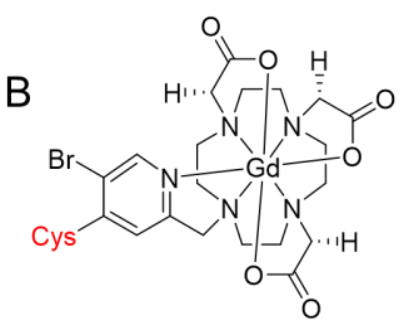

BrPy-DO3A-Gd(III)

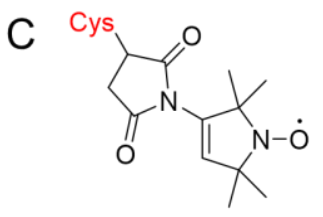

3-maleimido-PROXYL

Fig. S1. Spin labels used in the study. (A) MTSL, (B) BrPy-DO3A-Gd(III) and (C) 3-maleimidoPROXYL bound to the protein (Sba1 or Hp90) via a cysteine residue (indicated in red letters).

\section{Additional data on Sba1}

A

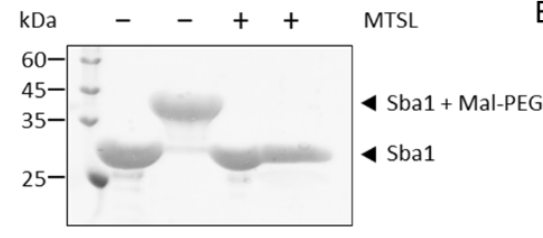

B

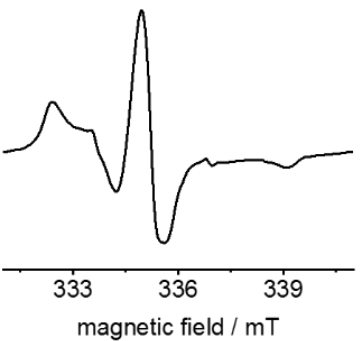

Fig. S2. Characterization of Sba1. (A) SDS-PAGE on Sba1 and Sba1 MTSL in absence and presence of Mal-PEG(5000) (Sigma-Aldrich). Sba1 is $26 \mathrm{kDa}$, whereas the Mal-PEG used has a molecular weight of $5 \mathrm{kDa}$. The presence of a band of $>26 \mathrm{kDa}$ in the sample of Sba1+Mal-PEG means the native cysteine is accessible to labeling. The absence of this high molecular weight band in the sample of Sba1/NO+Mal-PEG means Sba1 was quantitatively labeled by MTSL. Conditions: $12 \%$ acrylamide, $200 \mathrm{~V}$. (B) Room-temperature CW-EPR spectrum of Sba1/NO+WT yHsp90+Mn(II)·AMP-PNP 0.31/0.29/4 mM. Measurement parameters: microwave field: 9.4215 $\mathrm{GHz}$, attenuation: $10 \mathrm{~dB}$, modulation amplitude: $1 \mathrm{G}$, modulation frequency: $100 \mathrm{kHz}$, number of scans: 9. 


\section{DEER experimental parameters}

Table S2. Parameters of the NO-NO DEERs on samples with Sba1/NO.

\begin{tabular}{|l|l|l|l|}
\hline Sample & $\begin{array}{l}\text { Sba1/NO } \\
+\mathrm{Mg}(\mathrm{II}) \cdot \mathrm{AMP}-\mathrm{PNP}\end{array}$ & $\begin{array}{l}\text { WT+ Sba1/NO } \\
+\mathrm{Mg}(\mathrm{II}) \cdot \mathrm{AMP}-\mathrm{PNP}\end{array}$ & $\begin{array}{l}\text { WT+ Sba1/NO } \\
+\mathrm{Mg}(\mathrm{II}) \cdot \mathrm{ADP}\end{array}$ \\
\hline Shown in Fig. & S4, green & S4, black & S4, blue \\
\hline Experiment & DEER & DEER & DEER \\
\hline Temperature / K & 25 & 25 & 25 \\
\hline$\pi / 2, \pi$ pulse length / ns & 20,40 & 25,50 & 30,60 \\
\hline$\tau_{1} / \mu \mathrm{s}$ & 0.6 & 0.6 & 0.6 \\
\hline$\tau_{2} / \mu \mathrm{s}$ & 4 & 4 & 4 \\
\hline Pump pulse length / ns & 128 & 128 & 128 \\
\hline Starting t / ns & -200 & -200 & -200 \\
\hline Step / ns & 20 & 20 & 20 \\
\hline Repetition time / ms & 8 & 8 & 8 \\
\hline$v_{\text {obs }, v_{\text {pump }} / \mathrm{GHz}}$ & $94.85,94.9-95.0$ & $94.85,94.9-95.0$ & $94.85,94.9-95.0$ \\
\hline Measurement time / h & 23.5 & 20 & 38 \\
\hline
\end{tabular}

Table S3. Parameters of the Mn(II)-NO DEERs on samples with Sba1/NO.

\begin{tabular}{|l|l|l|l|}
\hline Sample & $\begin{array}{l}\text { WT+Sba1/NO } \\
+\mathrm{Mn}(\mathrm{II}) \cdot A M P-P N P\end{array}$ & $\begin{array}{l}\text { WT+Sba1/NO } \\
+\mathrm{Mn}(\mathrm{II}) \cdot \mathrm{ADP}\end{array}$ & $\begin{array}{l}\text { WT+Sba1/NO } \\
+ \text { Mn(II) ATP, 0.5 h }\end{array}$ \\
\hline Shown in Fig. & $1 \mathrm{C}$ & $1 \mathrm{C}$ & S5 \\
\hline Experiment & DEER & DEER & DEER \\
\hline Temperature / K & 10 & 2 & 10 \\
\hline$\pi / 2, \pi$ pulse length / ns & 30,60 & 20,40 & 20,40 \\
\hline$\tau_{1} / \mu \mathrm{s}$ & 0.6 & 0.5 & 0.6 \\
\hline$\tau_{2} / \mu \mathrm{s}$ & 3.1 & 4.5 & 3.6 \\
\hline Pump pulse length / ns & 128 & 128 & 128 \\
\hline Starting t / ns & -200 & -300 & -200 \\
\hline Step / ns & 20 & 20 & 20 \\
\hline Repetition time / ms & 0.8 & 0.7 & 0.4 \\
\hline$v_{\text {obs }} v_{\text {pump }} / \mathrm{GHz}$ & $95.08,94.75-94.95$ & $95.03,94.835-94.965$ & $95.05,94.75-94.95$ \\
\hline Measurement time / h & 12 & 22 & 3.5 \\
\hline
\end{tabular}


Table S4. Parameters of the Gd(III)-Gd(III) DEERs.

\begin{tabular}{|l|l|l|}
\hline Sample & $\begin{array}{l}\text { D560C/Gd(III)+Mg(II) AMP- } \\
\text { PNP } \pm \text { Sba1 }\end{array}$ & $\begin{array}{l}\text { K637C/Gd(III)+Mg(II) AMP- } \\
\text { PNP } \pm \text { Sba1 }\end{array}$ \\
\hline Shown in Fig. & S7 & S7 \\
\hline Experiment & DEER & DEER \\
\hline Temperature / K & 10 & 10 \\
\hline$\pi / 2, \pi$ pulse length / ns & 15,30 & 15,30 \\
\hline$\tau_{1} / \mu$ s & 0.6 & 0.6 \\
\hline$\tau_{2} / \mu$ s & 7.5 & 5.7 \\
\hline Pump pulse length / ns & 96,96 & 96,96 \\
\hline Starting t / ns & -200 & -200 \\
\hline Step / ns & 30 & 25 \\
\hline Repetition time / ms & 0.2 & 0.2 \\
\hline$v_{\text {obs }} v_{\text {pump }} / \mathrm{GHz}$ & $94.9,94.5-94.8$ and 95.0-95.3 & $94.9,94.5-94.8$ and 95.0-95.3 \\
\hline Measurement time / $h$ & $3.5(+$ Sba1) & 1.5 (-Sba1), 18 (+ Sba1) \\
\hline
\end{tabular}

Table S5. Parameters of the NO-NO DEERs on samples with A152C/NO.

\begin{tabular}{|l|l|l|}
\hline Sample & A152C/NO+Mg(II) AMP-PNP $*$ & A152C/NO+Mg(II) AMP-PNP+Sba1 \\
\hline Shown in Fig. & $2 \mathrm{~B}$ & $2 \mathrm{~B}$ \\
\hline Experiment & $r$ DEER & DEER \\
\hline Temperature / K & 25 & 25 \\
\hline$\pi / 2, \pi$ pulse length / ns & 30,60 & 30,60 \\
\hline$\tau_{1} / \mu \mathrm{s}$ & 6.2 & 0.2 \\
\hline$\tau_{2} / \mu \mathrm{s}$ & 2 & 5.7 \\
\hline Pump pulse length / ns & 128 & 128 \\
\hline Starting t/ns & -200 & -200 \\
\hline Step / ns & 30 & 25 \\
\hline Repetition time / ms & 20 & 20 \\
\hline$v_{\text {obs }} v_{\text {pump }} / \mathrm{GHz}$ & $94.85,94.9-95.05$ & $94.85,94.9-95.05$ \\
\hline Measurement time / $\mathrm{h}$ & 5.5 & 1.5 \\
\hline
\end{tabular}

$* *$ data previously published in ref $^{1}$ 
Table S6. Parameters of the Mn(II)-NO DEERs on samples with A152C/NO.

\begin{tabular}{|c|c|c|c|}
\hline Sample & $\begin{array}{l}\text { A152C/NO+ } \\
\text { Mn(II)·AMP-PNP }( \pm \text { Sba1 }) \\
\text { and A152C/NO+ } \\
\text { Mn(II)·ADP-Sba1 }\end{array}$ & $\begin{array}{l}\text { A152C/NO+ } \\
\text { Mn(II)·ADP-Sba1 }\end{array}$ & $\begin{array}{l}\mathrm{A} 152 \mathrm{C} / \mathrm{NO}+ \\
\mathrm{Mn}(\mathrm{II}) \cdot \mathrm{ADP}+\mathrm{Sba} 1\end{array}$ \\
\hline Shown in Fig. & 2D, S9 (rep) & S9 (\#2) & 2D, S9 (\#2) \\
\hline Experiment & DEER & DEER & DEER \\
\hline Temperature / K & 10 & 10 & 10 \\
\hline$\pi / 2, \pi$ pulse length $/ \mathrm{ns}$ & 30,60 & 30,60 & 20,40 \\
\hline$\tau_{1} / \mu \mathrm{s}$ & 0.6 & 0.6 & 0.6 \\
\hline$\tau_{2} / \mu \mathrm{s}$ & 3.1 & 3.8 & 3.5 \\
\hline Pump pulse length / ns & 128 & 128 & 128 \\
\hline Starting $\mathrm{t} / \mathrm{ns}$ & -200 & -200 & -200 \\
\hline Step / ns & 20 & 25 & 10 \\
\hline Repetition time / ms & 0.8 & 0.8 & 0.2 \\
\hline$v_{\text {obs }}, v_{\text {pump }} / \mathrm{GHz}$ & $95.1,94.75-94.95$ & 95.05 & $\begin{array}{l}95.1,94.75-94.95(3 \mathrm{~d}) \\
95.07,94.75-94.95(\# 2)\end{array}$ \\
\hline Measurement time / h & $\begin{array}{l}9 \text { (AMP·PNP-Sba1) } \\
18 \text { (AMP·PNP+Sba1) } \\
25 \text { (ADP-Sba1) }\end{array}$ & 1 & 25 (2D), $10.5(\# 2)$ \\
\hline
\end{tabular}


Table S7. Parameters of the Mn(II)-Mn(II) DEERs.

\begin{tabular}{|c|c|c|c|c|}
\hline Sample & $\begin{array}{l}\text { WT+ } \\
\text { Mn(II)·AMP } \\
\text {-PNP+Sba1 }\end{array}$ & $\begin{array}{l}\text { A152C+ } \\
\text { Mn(II) AMP- } \\
\text { PNP+Sba1 }\end{array}$ & $\begin{array}{l}\text { WT+ } \\
\text { Mn(II)·ADP+ } \\
\text { Sba1 }\end{array}$ & $\begin{array}{l}\text { A152C+ } \\
\text { Mn(II) ADP+ } \\
\text { Sba1 }\end{array}$ \\
\hline Shown in Fig. & $3 \mathrm{~B}$ & S8 & $3 \mathrm{~B}$ & S8 \\
\hline Experiment & $r$ DEER & DEER & $r$ DEER & $r$ DEER \\
\hline Temperature / K & 10 & 10 & 10 & 10 \\
\hline$\pi / 2, \pi$ pulse length $/ \mathrm{ns}$ & 15,30 & 15,30 & 15,30 & 15,30 \\
\hline$\tau_{1} / \mu \mathrm{s}$ & 3.8 & 0.6 & 3.7 & 3.0 \\
\hline$\tau_{2} / \mu \mathrm{s}$ & 3 & 3.1 & 2.5 & 2.5 \\
\hline Pump pulse length / ns & 96,96 & 136 & 96,96 & 96,96 \\
\hline Starting $\mathrm{t} / \mathrm{ns}$ & -200 & -200 & -200 & -200 \\
\hline Step / ns & 20 & 20 & 15 & 15 \\
\hline Repetition time / ms & 0.8 & 0.2 & 0.8 & 0.8 \\
\hline$v_{\text {obs }}, v_{\text {pump }} / \mathrm{GHz}$ & $\begin{array}{l}94.9, \quad 94.5- \\
94.8 \quad \text { and } \\
95.0-95.3\end{array}$ & $\begin{array}{l}94.85, \\
94.925- \\
95.075\end{array}$ & $\begin{array}{l}94.9, \quad 94.5- \\
94.8 \text { and } 95.0- \\
95.3\end{array}$ & $\begin{array}{l}94.9, \quad 94.5- \\
94.8 \text { and 95.0- } \\
95.3\end{array}$ \\
\hline Measurement time / $\mathrm{h}$ & 11.5 & 11.5 & 5 & 2.5 \\
\hline
\end{tabular}




\section{DEER experimental set-up}
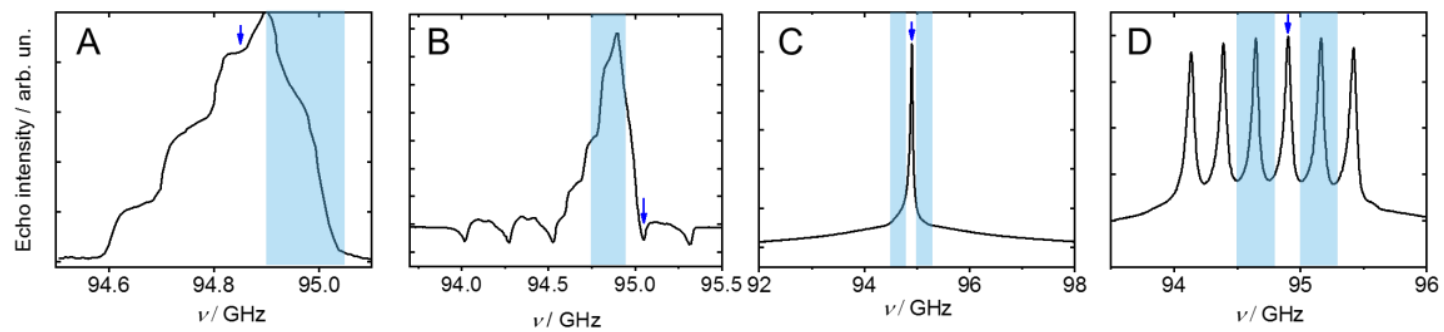

Fig. S3. W-band ED-EPR spectra of the different spin labels. (A) NO spins (25 K), (B) Mn(II) and NO spins optimized for the NO spins (10 K), (C) Gd(III) spins (10 K) and (D) Mn(II) spins only $(10 \mathrm{~K})$. The blue arrow and shaded area show the general experimental set-up and correspond to the positions of the observe and pump pulses, for measuring NO-NO, Mn(II)-NO, Gd(III)-Gd(III), and $\mathrm{Mn}(\mathrm{II})-\mathrm{Mn}(\mathrm{II}) \mathrm{DEER}$, respectively. The samples are $\mathrm{A} 152 \mathrm{C} / \mathrm{NO}+\mathrm{Mg}(\mathrm{II}) \cdot \mathrm{ADP}$ (A), A152C/NO+Mn(II) AMP-PNP (B), D560C/Gd(III) in apo state (C), and WT+Mn(II) AMP-PNP (D). For exact pump-observe positions see Tables S2-S7. 


\section{Additional DEER data}

\section{NO-NO DEER on Sba1/NO in presence and absence of Sba1}

To establish Sba1 binding to yHsp90 we started with the obvious choice of singly labeling Sba1 with MTSL (structure shown in Fig. S1A), referred to as Sba1/NO, and measured NO-NO DEER in the presence of yHsp90. Here we expect to see a DEER effect only if Sba1 is bound to both protomers. We, initially, performed the experiment in the presence of AMP-PNP as it is known from literature that Sba1 binds in the pre-hydrolysis state ${ }^{13-15}$. The NO-NO DEER measurements (set-up and data are in Fig. S3A and S4, respectively) on yHsp90 in the presence of Sba1/NO gave a modest DEER effect with a modulation depth, $\lambda$, of $\sim 2-3 \%$ as opposed to $12 \%$ expected for $100 \%$ binding of two Sba1 molecules under our experimental conditions. Moreover, the distance distribution showed a peak around $3.4 \mathrm{~nm}$ and some intensity around $5 \mathrm{~nm}$ with high uncertainty, whereas the predicted NO-NO distance from the X-ray structure is $4.6 \mathrm{~nm}^{11}$. We carried out similar measurements with $\mathrm{Mg}(\mathrm{II}) \cdot \mathrm{ADP}$ and obtained similar results (Fig. S4). We also carried out control measurements on samples containing only Sba1 and excess of $\mathrm{Mg}(\mathrm{II}) \cdot \mathrm{AMP}-\mathrm{PNP}$ to check for the possible presence of Sba1 dimers that may interfere with the observed signals. Indeed, also here, we recovered a DEER trace with $\lambda$ of $2 \%$ (Fig. S4) with a distance distribution similar to that observed with yHsp90 indicating the presence of a small percentage of dimers with a NO-NO distance at $3.4 \mathrm{~nm}$ further complicating interpretation of data in presence of yHsp90. While Sba1 has been found to be monomeric in solution (with ultra-centrifugation), the dimeric form has also been found with X-ray crystallography for the human homologue (p23) via disulfide bond formation of the $\mathrm{C} 58^{16}$. The authors stated that the dimer is 'either a minor form of the protein or an artifact of crystallization' ${ }^{16}$. Additionally, Hsp90 dimers have been previously observed in SDS page $^{15}$. The NO-NO DEER results described above are inconclusive in terms of determination of the Sba1/yHsp90 complex formation due to the Sba1 dimer's interference. 

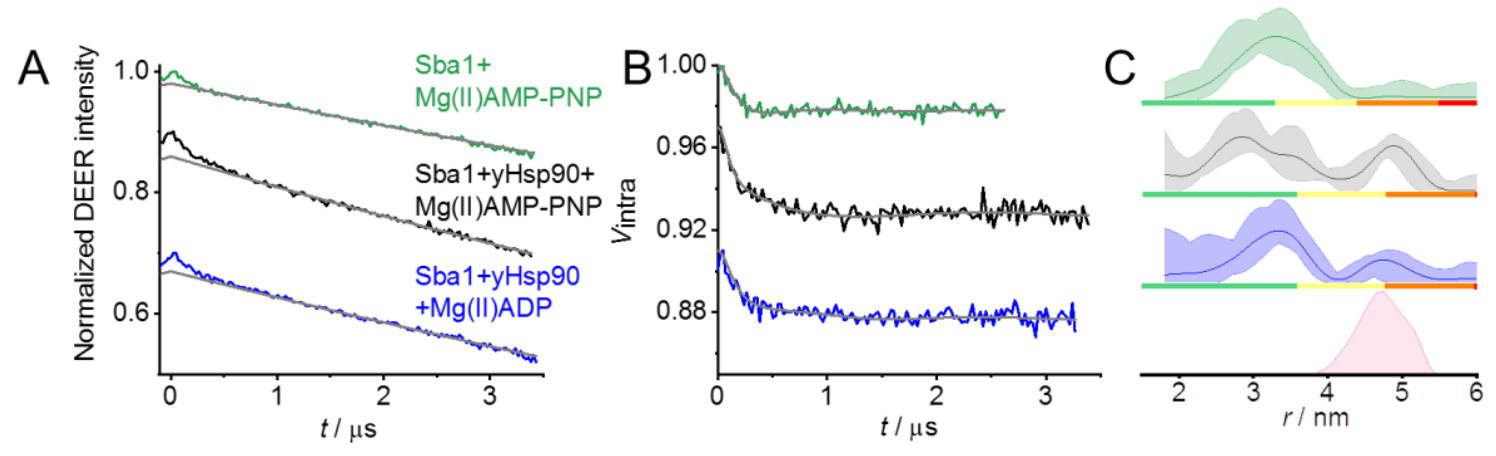

Fig. S4. W-band NO-NO DEER data on Sba1/NO in absence and presence of yHsp90. (A) Primary data with the gray line indicating the background decay. (B) Background-corrected DEER data with the gray line being the fit to the data. (C) Distance distributions with confidence intervals, showing also the modeled NO-NO distance distribution (in pink color) for Sba1/NO bound to each protomer. The samples are in indicated in (A), the experimental set-up and sample composition are in Fig. S3A and Table S1, respectively. The color bars on the distance distributions denote reliability as follows: green $=$ shape reliable; yellow $=$ mean and width reliable; orange $=$ mean reliable; red= non-reliable. The DEER trace in absence of yHsp90 is cut due to an artifact. 

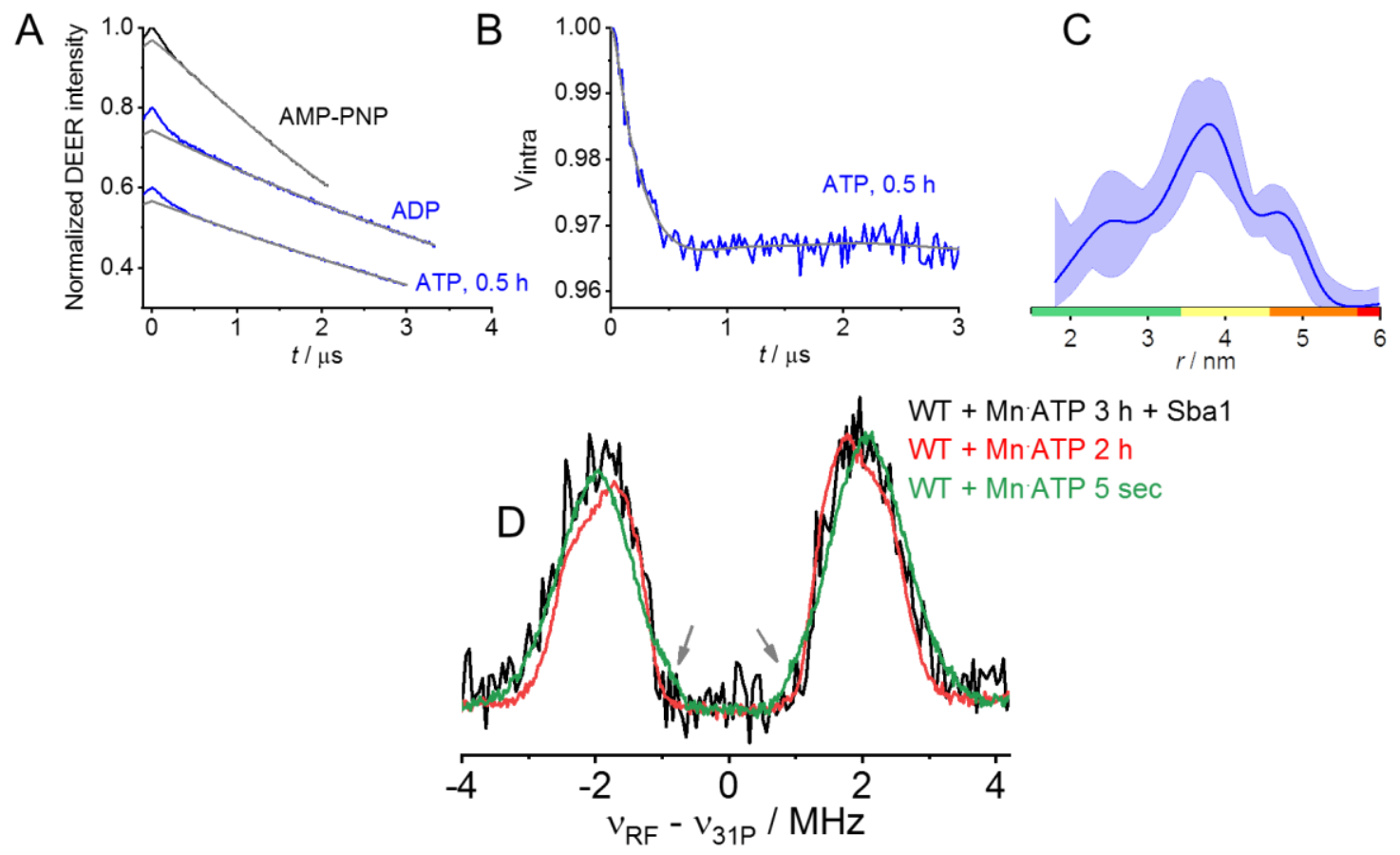

Fig. S5. Additional Mn(II)-NO DEER data of Fig. 1 and ${ }^{31}$ P ENDOR in presence Sba1. (A) Primary DEER data with the gray lines indicating the background decay. (B) Background-corrected DEER data and (C) distance distribution with confidence intervals as defined in Fig. S4 in presence of Mn(II) ATP after hydrolysis has occcured. (D) ${ }^{31}$ P Davies ENDOR in presence of Sba1 and ATP, $3 \mathrm{~h}$ reaction time (black) and comparison with samples in absence of Sba1 in the pre- (green) and post- (red) hydrolysis states. The spectra in red and green color were previously published in ref ${ }^{1}$. The arrows point to spectral features typical of Mn(II) ATP/AMP-PNP ${ }^{17}$. 


\section{Control experiments}

First, we performed a Mn(II)-Mn(II) DEER measurement by moving the field to the Mn(II) spectral region outside the NO spectrum for the yHsp90+Sba1/NO+Mn(II)-AMP-PNP sample under the same experimental set-up, optimized for NO pumping, and no DEER effect was observed (Fig. S6A). Also, we prepared a sample that has the same composition as above but without yHsp90, i.e. Sba1/NO+Mn(II) AMP-PNP, and performed Mn(II)-NO DEER under the same experimental conditions and again no DEER modulation was observed (Fig. S6B). We additionally excluded the option of observing $\mathrm{Mn}$ (II)-Mn(II) distance as with our set-up and spectrometer conditions we obtain a $\lambda$ of $0.5 \%$ for the $\mathrm{Mn}(\mathrm{II})-\mathrm{Mn}$ (II) distance in presence of AMP-PNP and absence of Sba1 (the respective value was $1.8 \%$ in presence of $\mathrm{ADP})^{1}$. Therefore, we exclude the possibility to observe $\mathrm{Mn}(\mathrm{II})-\mathrm{Mn}$ (II) distance with a $\lambda$ of $3 \%$ and (5\% for the post-hydrolysis state). Moreover, we exclude the possibility that the observed distances come from NO-NO interaction (i.e. between the two Sba1/NO bound to each protomer) because the experiment was performed under conditions optimized for observing $\mathrm{Mn}$ (II) spins, i.e. at $10 \mathrm{~K}$ and with a fast repetition time ( $<1 \mathrm{~ms})$. These control measurements confirm that the observed DEER effect comes from hetero-pairs between Mn(II) on yHsp90 and NO on Sba1.
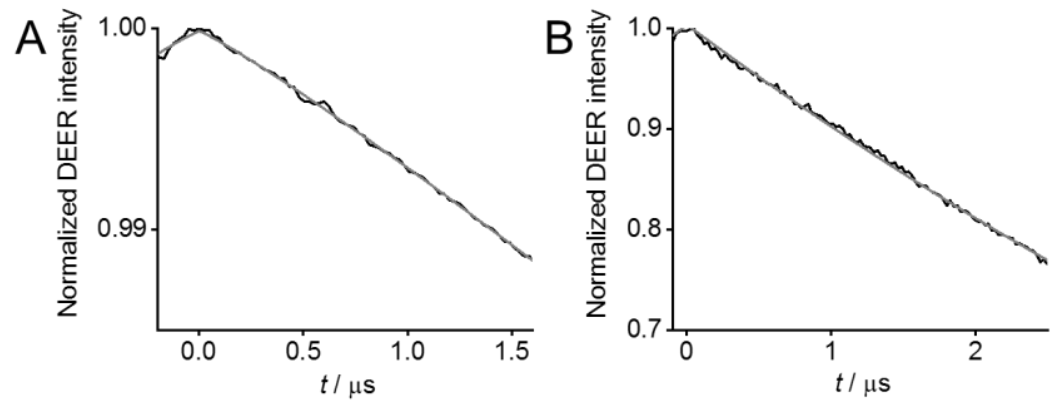

Fig. S6. Control DEER measurements. (A) Primary Mn(II)-Mn(II) DEER under NO pumping conditions in sample yHsp90(WT)+Sba1/NO+Mn(II) AMP-PNP. (B) Primary Mn(II)-NO DEER of Sba1/NO+Mn(II)-AMP-PNP, i.e. as in (A) but without WT yHsp90. The gray lines indicate the background decay. 

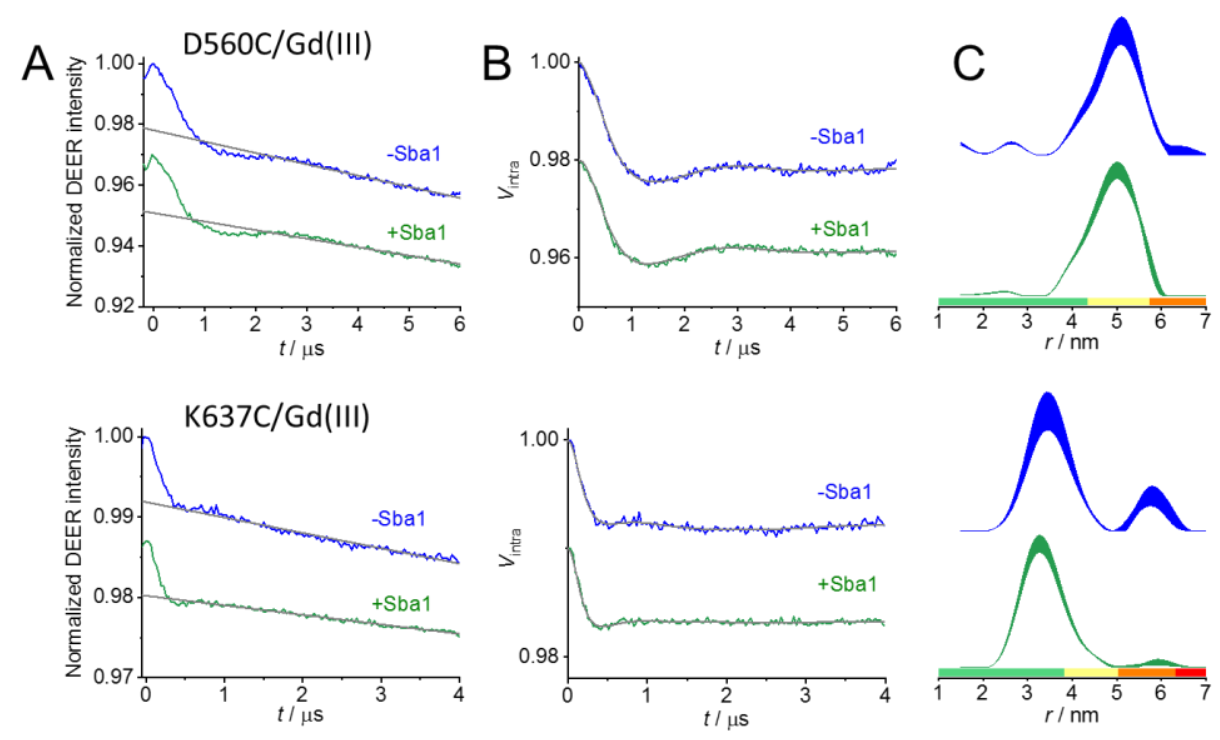

Fig. S7. W-band Gd(III)-Gd(III) DEER data on D560C/Gd(III) and K637C/Gd(III) in absence and presence of non-labeled Sbal and excess of Mg(II) AMP-PNP. (A) Primary data with the gray line indicating the background decay (experimental set-up in Fig. S3C). (B) Background-corrected DEER data with the gray line being the fit to the data. (C) Distance distributions with confidence intervals. The color bars below the distance distributions denote reliability as defined in Fig. S4.
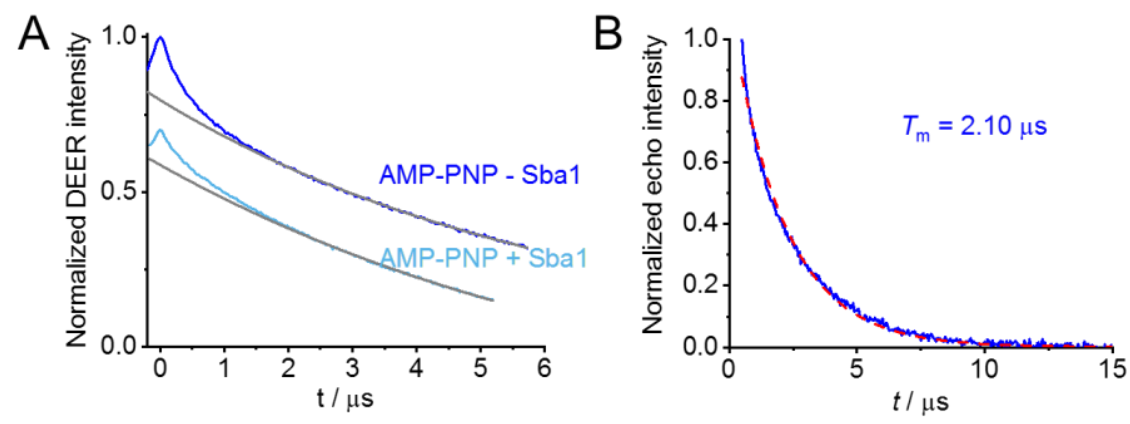

Fig. S8. Additional EPR data of Fig. 2B. (A) Primary NO-NO DEER data with the gray line indicating the background decay. (B) Echo decay trace of sample in A152C/NO+Mg(II) AMP-PNP in absence of Sba1 with the $T_{\mathrm{m}}$, the phase memory time, as found from fitting the data to a monoexponential fit (in red dashed line). 
A
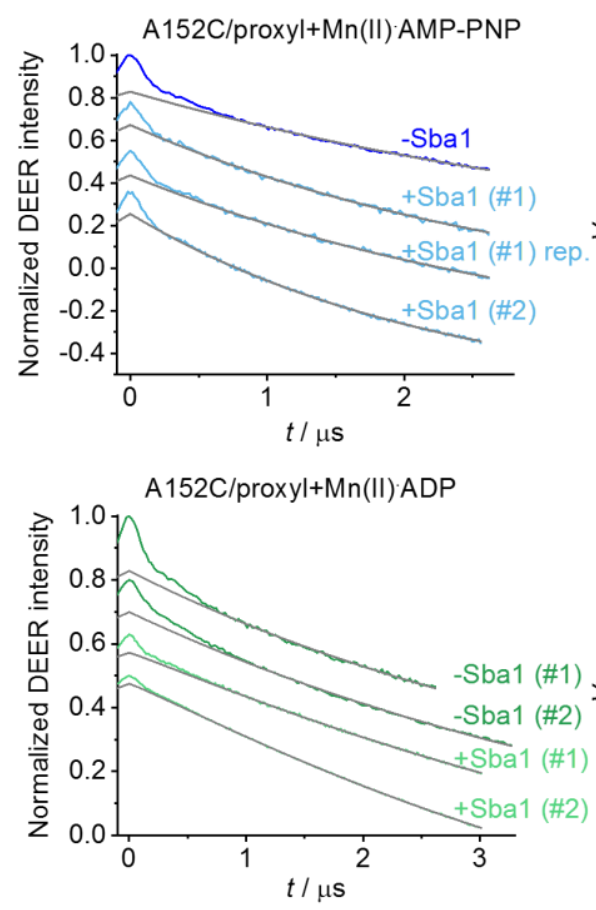

B
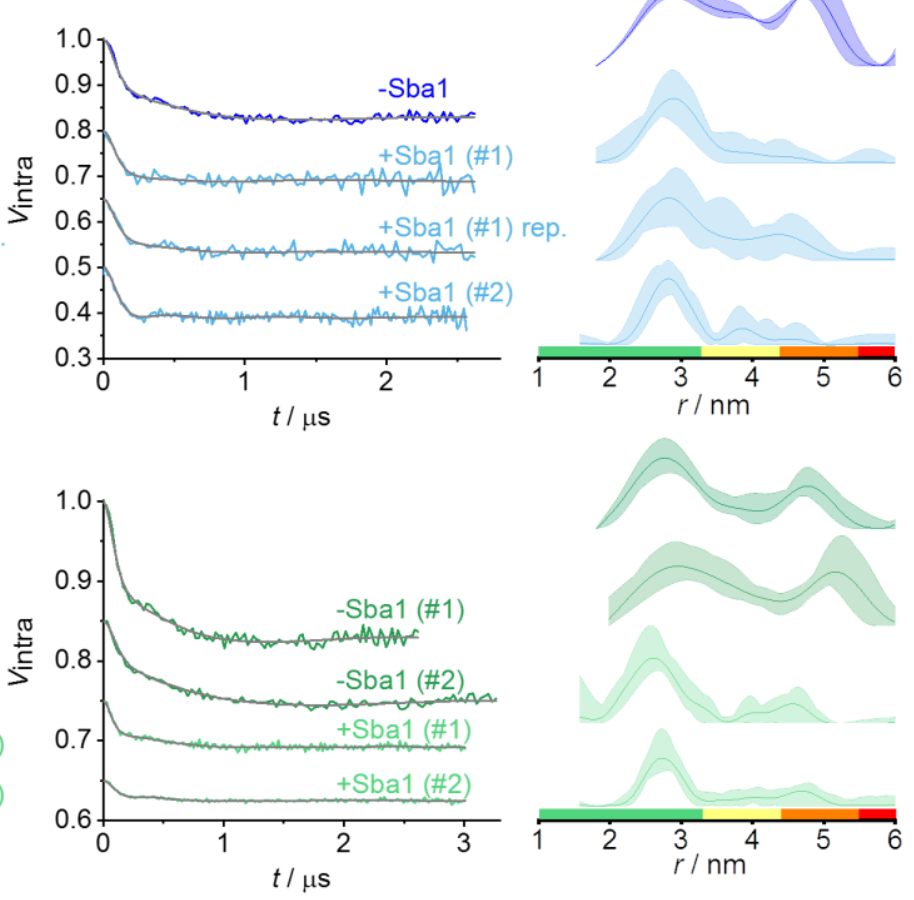

Fig. S9. W-band Mn(II)-NO DEER data in absence and presence of Sba1 and sub-stoichiometric amounts of Mn(II) AMP-PNP (top) and Mn(II) ADP (bottom) for mutant A152C/NO. (A-C) As defined in Fig. S7 with the color bars below the distance distributions denoting reliability as defined in Fig. S4 and calculated for the shortest DEER trace. The different sample preparations are denoted with \#, whereas measurement repeats are denote as 'rep.' 

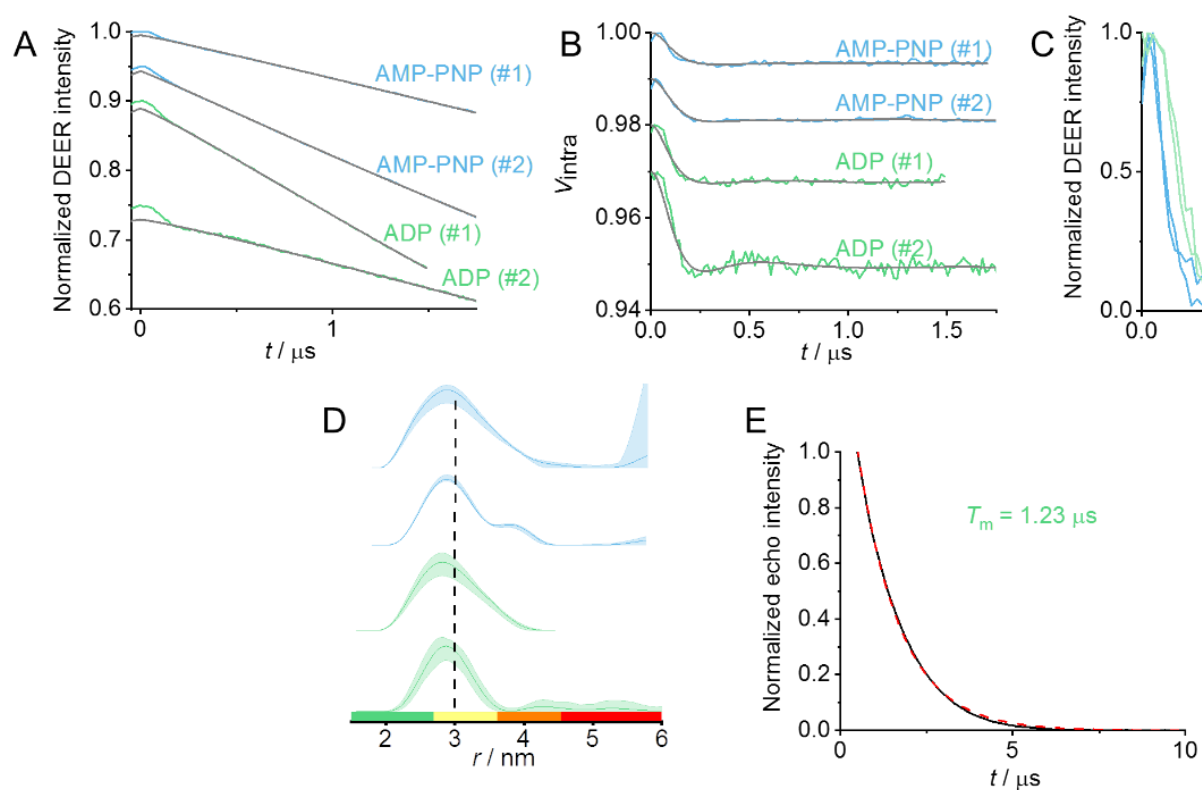

Fig. S10. W-band Mn(II)-Mn(II) DEER data in presence of Sba1 and sub-stoichiometric amounts of Mn(II)-AMP-PNP (cyan color) and Mn(II)-ADP (light green color) for WT yHsp90. (A, B, D) As defined in Fig. S7 with the addition of the normalized DEER traces (C). The color bars below the distance distributions denote reliability as defined in Fig. S4 and calculated for the shortest DEER trace. The sample denoted as \#2 are non-labeled A152C mutant. (E) Echo decay trace of $\mathrm{Mn}$ (II) spins in sample in $\mathrm{A} 152 \mathrm{C}\left(\right.$ non-labeled)+Mn(II) $\mathrm{ADP}+\mathrm{Sba} 1$ with the $T_{\mathrm{m}}$ as found from fitting the data to a mono-exponential fit (in red dashed line). 


\section{References}

(1) Giannoulis, A.; Feintuch, A.; Barak, Y.; Mazal, H.; Albeck, S.; Unger, T.; Yang, F.; Su, X. C.; Goldfarb, D. Two Closed ATP- and ADP-Dependent Conformations in Yeast Hsp90 Chaperone Detected by Mn(II) EPR Spectroscopic Techniques. Proc. Natl. Acad. Sci. U.S.A. 2020, 117, 395404.

(2) Goldfarb, D.; Lipkin, Y.; Potapov, A.; Gorodetsky, Y.; Epel, B.; Raitsimring, A. M.; Radoul, M.; Kaminker, I. HYSCORE and DEER with an Upgraded 95GHz Pulse EPR Spectrometer. $J$. Magn. Reson. 2008, 194, 8-15.

(3) Feher, G. Observation of Nuclear Magnetic Resonances via the Electron Spin Resonance Line. Phys. Rev. 1956, 103, 834-835.

(4) Mentink-Vigier, F.; Collauto, A.; Feintuch, A.; Kaminker, I.; Le, V. T.; Goldfarb, D. Increasing Sensitivity of Pulse EPR Experiments Using Echo Train Detection Schemes. J. Magn. Reson. 2013, 236, 117-125.

(5) Epel, B.; Arieli, D.; Baute, D.; Goldfarb, D. Improving W-Band Pulsed ENDOR SensitivityRandom Acquisition and Pulsed Special Triple. J. Magn. Reson. 2003, 164, 78-83.

(6) Bahrenberg, T.; Yang, Y.; Goldfarb, D.; Feintuch, A. rDEER: A Modified DEER Sequence for Distance Measurements Using Shaped Pulses. Magnetochemistry 2019, 5, $20-34$.

(7) Pannier, M.; Veit, S.; Godt, A.; Jeschke, G.; Spiess, H. W. Dead-Time Free Measurement of Dipole-Dipole Interactions between Electron Spins. J. Magn. Reson. 2000, 142, 331-340.

(8) Bahrenberg, T.; Rosenski, Y.; Carmieli, R.; Zibzener, K.; Qi, M.; Frydman, V.; Godt, A.; Goldfarb, D.; Feintuch, A. Improved Sensitivity for W-Band Gd(III)-Gd(III) and NitroxideNitroxide DEER Measurements with Shaped Pulses. J. Magn. Reson. 2017, 283, 1-13.

(9) Jeschke, G.; Chechik, V.; Ionita, P.; Godt, A.; Zimmermann, H.; Banham, J.; Timmel, C. R.; Hilger, D.; Jung, H. DeerAnalysis2006 - a Comprehensive Software Package for Analyzing Pulsed ELDOR Data. Appl. Magn. Reson. 2006, 30, 473-498.

(10) Hagelueken, G.; Ward, R.; Naismith, J. H.; Schiemann, O. MtsslWizard: In Silico SpinLabeling and Generation of Distance Distributions in PyMOL. Appl. Magn. Reson. 2012, 42, 377391.

(11) Ali, M. M.; Roe, S. M.; Vaughan, C. K.; Meyer, P.; Panaretou, B.; Piper, P. W.; Prodromou, C.; Pearl, L. H. Crystal Structure of an Hsp90-Nucleotide-p23/Sba1 Closed Chaperone Complex. Nature 2006, 440, 1013-1017.

(12) Verba, K. A.; Wang, R. Y.; Arakawa, A.; Liu, Y.; Shirouzu, M.; Yokoyama, S.; Agard, D. A. Atomic Structure of Hsp90-Cdc37-Cdk4 Reveals That Hsp90 Traps and Stabilizes an Unfolded Kinase. Science 2016, 352, 1542-1547.

(13) McLaughlin, S. H.; Sobott, F.; Yaol, Z. P.; Zhang, W.; Nielsen, P. R.; Grossmann, J. G.; Laue, E. D.; Robinson, C. V.; Jackson, S. E. The Co-Chaperone p23 Arrests the Hsp90 ATPase Cycle to Trap Client Proteins. J. Mol. Biol. 2006, 356, 746-758.

(14) Siligardi, G.; Hu, B.; Panaretou, B.; Piper, P. W.; Pearl, L. H.; Prodromou, C. Co-Chaperone Regulation of Conformational Switching in the Hsp90 ATPase Cycle. J. Biol. Chem. 2004, 279, 51989-51998.

(15) Richter, K.; Walter, S.; Buchner, J. The Co-Chaperone Sba1 Connects the ATPase Reaction of Hsp90 to the Progression of the Chaperone Cycle. J. Mol. Biol. 2004, 342, 1403-1413.

(16) Weaver, A. J.; Sullivan, W. P.; Felts, S. J.; Owen, B. A.; Toft, D. O. Crystal Structure and Activity of Human p23, a Heat Shock Protein 90 Co-Chaperone. J. Biol. Chem. 2000, 275, 2304523052.

(17) Litvinov, A.; Feintuch, A.; Un, S.; Goldfarb, D. Triple Resonance EPR Spectroscopy Determines the $\mathrm{Mn}^{2+}$ Coordination to ATP. J. Magn. Reson. 2018, 294, 143-152. 